\title{
Comparative Study Between Porcine Small Intestinal Submucosa and Buccal Mucosa in a Partial Urethra Substitution in Rabbits
}

\author{
Paulo Roberto Kawano, M.D., Oscar Eduardo Hidetoshi Fugita, M.D., ${ }^{1}$ Hamilto Akihissa Yamamoto, M.D., \\ Juliany Gomes Quitzan, M.D.,' Carlos Padovani, Ph.D., and João Luiz Amaro, M.D.'
}

\begin{abstract}
Purpose: Several urethral conditions may require tissue substitution. One collagen-base biomaterial that recently emerged as an option is small intestinal submucosa (SIS). The aim of this study was to compare the results of SIS and buccal mucosa for urethral substitution in rabbits.

Materials and Methods: Thirty-six North Folk male rabbits were randomized into three groups. In all animals, a $10 \times 5 \mathrm{~mm}$ urethral segment was excised, and the urethral defect was repaired using a one-layer SIS patch (group I [GI]); four-layer SIS (group II [GII]); or buccal mucosa (group III [GIII]). Urethrography was performed preoperatively and after 12 weeks. After sacrifice, graft retraction was objectively measured using Scion Image ${ }^{\circledR}$ computer analysis and by calculation of ellipse area. The grade of fibrosis, inflammatory reaction, vascular/ epithelial regeneration, and collagen III/I ratio were analyzed by hematoxylin/eosin and Picrosirius red staining.

Results: Urethrography confirmed a wide urethral caliber without any signs of strictures after surgery. Urethral fistulae was diagnosed in $8.3 \%$ of cases (1 animal each group). Average graft shrinkage was $55.2 \%$ in GI; $44.2 \%$ in GII; and $57.2 \%$ in GIII $(p<0.05)$. The intensity of chronic inflammation, fibrosis, epithelium regeneration, and neovascularization was similar in all groups $(p>0.05)$. Collagen III/I ratio was higher in GII (GI: 119.6; GII: 257.2 and GIII: 115.0); $p<0.01$.

Conclusions: The four-layer SIS is more advantageous than the one-layer SIS and buccal mucosa for urethral substitution in rabbits.
\end{abstract}

\section{Introduction}

$\mathbf{S}$ URGERY FOR URETHRAL RECONSTRUCTION in patients presenting congenital or acquired lesions is challenging, and the improvements of the aesthetic and functional results are due, in part, to the development of new materials and techniques. ${ }^{1,2}$

In an attempt to get better results, numerous procedures have been proposed, including complete surgical excision of the affected urethral segment or partial replacement by autologous materials. ${ }^{2-4}$ However, even autologous materials may have unsatisfactory results if the conditions of the vascular bed adjacent to the receptor tissue are unfavorable; particularly in multi-operated patients. Furthermore, the use of nongenital tissues for urethroplasty is associated with additional procedures to obtain the graft, prolonging the surgical time, extending the hospital stay, and increasing the morbidity of the procedure. ${ }^{5}$
In order to avoid these problems, recent researches have been engaged in the search for non-self materials capable of providing adequate tissue replacement. ${ }^{6}$ The small intestinal submucosa (SIS) consists of a collagen-based, nonimmunogenic, xenogenic material harvested from the submucosal layer of porcine intestine. It has been used in urology as a partial substitute in bladder, ureter, and urethra augmentation. ${ }^{7-9}$ This material has been reported as safe and technically easy to handle, having the advantage of not requiring an additional incision in the patient. ${ }^{10}$

In order to search for an ideal substitute material for the reconstructive surgery of the urethra, we conducted a randomized comparative study to determine whether there are differences in the histological response of the receptor urethral tissue to the SIS graft with one or four layers and compare them with the buccal mucosa during the partial replacement of the urethra in an experimental model in rabbits.

\footnotetext{
${ }^{1}$ Department of Urology, São Paulo State University—UNESP, Botucatu, Brazil.

${ }^{2}$ Department of Bioestatistics, São Paulo State University-UNESP, Botucatu, Brazil.
} 


\section{Materials and Methods}

A total of 36 male white North Folk rabbits weighing 3.2$4.7 \mathrm{~kg}$ (average weight $3.7 \mathrm{~kg}$ ) were assigned to three experimental groups: group 1 (GI; $n=12$ ) underwent an onlay urethroplasty with one-layer SIS graft; in group 2 (GII; $n=12)$, the urethroplasty was performed using a four-layer SIS graft; and in group 3 (GIII; $n=12$ ), the urethral defect was treated using a buccal mucosa graft obtained from the animal itself. The graft size for all groups was similar with $10 \times 5 \mathrm{~mm}$ length. Rabbits were anesthetized with pentobarbital sodium $(3.5 \%$ at doses of $1 \mathrm{mg} / \mathrm{kg}$ ) intravenously, in two moments: before surgery, when the first retrograde urethrogram was performed; and before sacrifice, to allow urine sample collection and to obtain the second radiologic image of the urethra. For the interpretation of radiological findings, we evaluated the presence of contrast extravasation and changes in luminal diameter of the urethra at the implant site (Fig. 1). Enrofloxacin was administered to all rabbits once a day for a total of 7 days.

The SIS graft was cut in an ellipsoid shape about $10 \mathrm{~mm}$ long and $5 \mathrm{~mm}$ width, and immersed in sterile saline solution for about 5 minutes before the surgical implant in order to hydrate it. To obtain the buccal mucosa graft, an elliptical shape fragment measuring $10 \times 5 \mathrm{~mm}$ was removed from the mucosal surface along the upper jaw.

Preoperatively, a $10 \mathrm{~F}$ transurethral catheter was placed in situ. The anterior urethra was dissected and incised longitudinally on its ventral surface, and a fragment of $5 \times 10 \mathrm{~mm}$ was excised. The SIS graft previously prepared with one or four layers (GI and GII) or buccal mucosa (GIII) was attached to the urethral defect using a running 7.0 polyglactin suture.

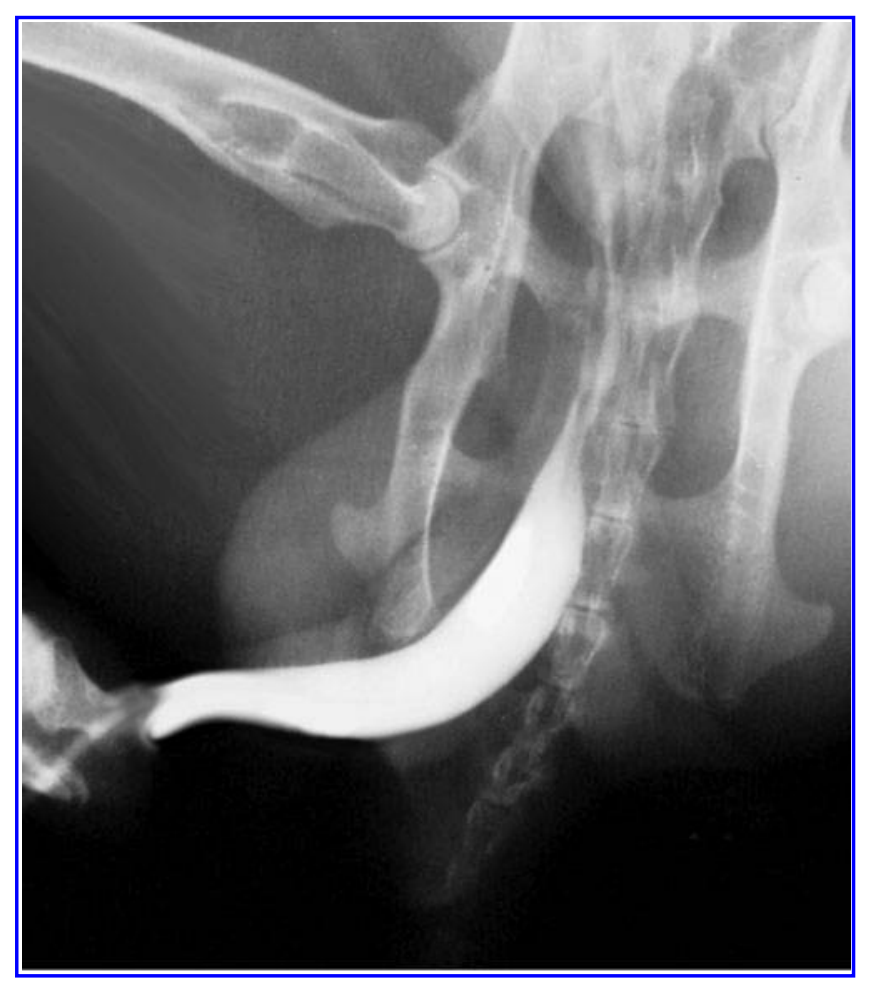

FIG. 1. Retrograde urethrogram demonstrating a normal urethra aspect.
The proximal, distal, and lateral edges of the graft were marked with a permanent suture (7.0 polypropylene) in order to identify the transition between the graft and normal urethral tissue. At the end of the procedure, saline solution was injected in the urethra to check the tightness of the suture and the patency of the urethra. The subcutaneous tissue was used to cover the graft, and the skin was reaproximated with 4.0 chromic sutures. No urethral catheter was left behind. Animals were sacrificed 12 weeks after surgery, and the penis was removed "en bloc." To assess the presence of fistulas, methylene blue was injected in an antegrade fashion in the urethra, while the distal end was maintained occluded. The penis was dissected, and the urethra was opened at the dorsal surface for adequate exposure of the graft (Fig. 2). The grafts were individually measured and photographed. The specimens were processed for routine histology.

Before implantation, the previously hydrated SIS and fresh buccal mucosa grafts were extended on a sterile plate and photographed. Their measurements were taken with a millimeter scaled ruler, where the length was considered the largest diameter $(2 a)$ and breadth, the lesser one $(2 b)$. The graft area was calculated by using the formula of the ellipse area: Area $=\pi \cdot a . b$.

After sacrifice, the graft area was measured again employing the methodology just mentioned. This entire process was conducted in a photographic studio using a professional digital camera at $2.5 \mathrm{~cm}$ standard focal length, and the images were analyzed by a software-Scion Image ${ }^{\circledR}$. After demarcation of the graft limits, data were automatically processed and calculated providing the final area in square millimeters. This process was easily performed with the aid of the nonabsorbable polypropylene stitches placed at the graft edges during the surgery. Formalin-fixed tissue samples were stained using the hematoxylin and eosin (HE) and evaluated in accordance with grade of fibrosis, inflammatory reaction, and vascular/ epithelial regeneration. These changes were classified as mild, moderate, or intense according to their characteristics when compared with surrounding tissue. Segments of normal

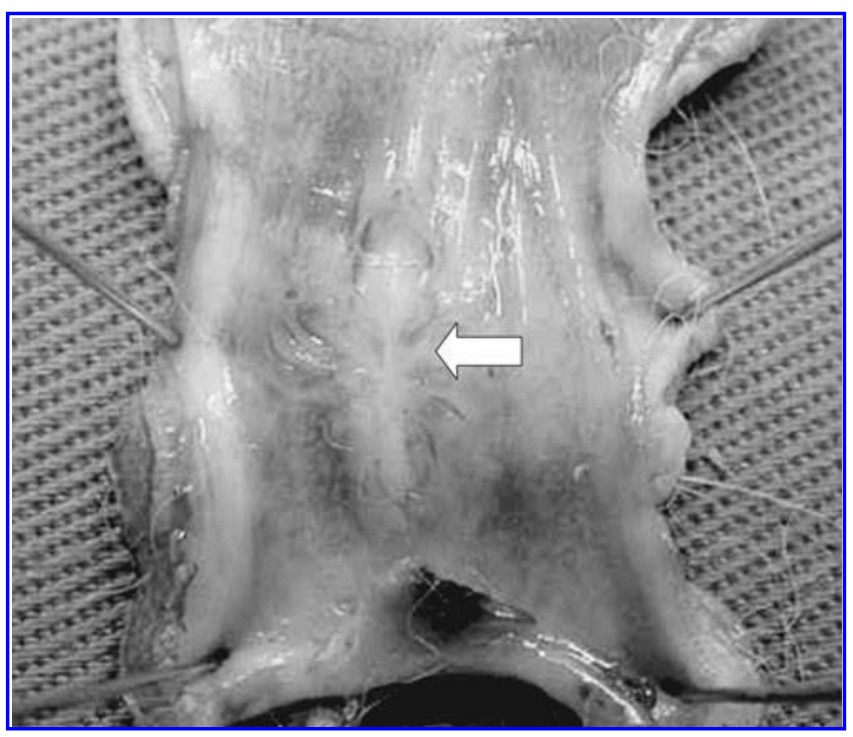

FIG. 2. Longitudinal opening of the urethra in its dorsal surface showing the graft area (arrow). 
Table 1. Median and Semi-Amplitude of Histological Parameters and Outcome of the Kruskal-Wallis Analysis of Variance by Study Group

\begin{tabular}{|c|c|c|c|c|}
\hline \multirow[b]{2}{*}{ Variable } & \multicolumn{3}{|c|}{ Group } & \multirow[b]{2}{*}{ Statistics } \\
\hline & $G I$ & G II & G III & \\
\hline Inflamatory infiltrate & $1.0 \pm 1.0$ & $1.0 \pm 1.0$ & $1.0 \pm 1.0$ & $0.00(p>0.005)$ \\
\hline Fibrosis & $3.0 \pm 0.5$ & $3.0 \pm 0.5$ & $2.0 \pm 0.5$ & $2.60(p>0.005)$ \\
\hline Epithelial regeneration & $2.0 \pm 1.0$ & $2.0 \pm 1.0$ & $2.0 \pm 1.0$ & $3.28(p>0.005)$ \\
\hline Neovascularization & $1.0 \pm 1.0$ & $1.0 \pm 1.0$ & $1.0 \pm 1.0$ & $0.61(p>0.005)$ \\
\hline
\end{tabular}

urethra and SIS were also processed in the same histological technique to evaluate the impact of surgical manipulation on the industrial product or primitive tissues.

For the quantification of the collagen fibers, the sections were stained with Picrosirius red. Four random fields were analyzed for each animal, and the surface area occupied by collagen fibers in each field was measured in micrometers using the Pro-Image Plus ${ }^{\circledR}$ morphometry program.

For statistical analysis of continuous variables, the Student's $t$-test was applied. For nominal variables, the Fischer's test was employed. Pearson correlation coefficient was used for calculation of urethral area, while profile analysis was used for comparison of groups according to the urethral area measurements. The determination of significance of histological parameters according to the study groups as well as comparisons between different collagen types in each sample was performed using Kruskal-Wallis analysis of variance. All statistical relevance was performed with a $0.05 \%$ significance level. The study methodology and techniques were approved by the Animal Experimentation Ethics Committee of the institution.

\section{Results}

None of the rabbits died before sacrifice. No animal had noticeable voiding dysfunction or stone formation during the study period. One rabbit in GI had an infection in the left

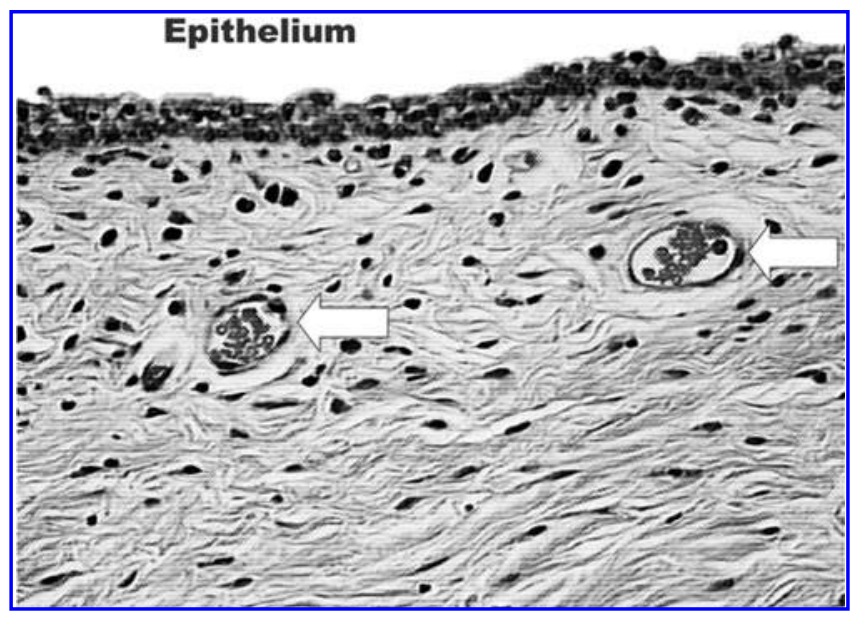

FIG. 3. Hematoxylin and Eosin stained section of the small intestinal submucosa graft area $(20 \times$ magnification) showing mild inflammatory infiltrate; delicate neoformed vessels (arrow); and good epithelial regeneration. testicle, which was treated medically. Two animals (GI and GIII) showed bacterial growth in urine samples, characterized by the identification of Enterococcus faecalis, but in both cases, the number of colonies was less than 100,000. The preoperative urethrography showed a wide urethral caliber without any signs of strictures in all animals, and the urethral area study did not show difference before and after surgery in all the groups. The incidence of urethral fistula was $8.3 \%$ (one animal per group). In all cases, the fistula orifices were visible.

Histological analysis performed by HE staining showed no significant difference $(p>0.05)$ with regard to the following parameters: fibrosis, epithelial regeneration, neovascularization, and inflammatory reaction (Table 1), (Fig. 3).

The evaluation of the conventional method (ellipse area) showed that the final graft area was significantly higher in GI $\left(21.52 \mathrm{~mm}^{2}\right)$ when compared with GIII $\left(13.74 \mathrm{~mm}^{2}\right)(p<0.05)$. However, the final area obtained from the scanned images analyzed by Scion Image Software showed a difference only between GII and GIII, where the graft areas were significantly smaller in the last one $\left(58.48 \mathrm{~mm}^{2}\right.$ vs. $44.8 \mathrm{~mm}^{2}$, respectively). So, regardless of the method used, the tissue retraction was greater among animals that underwent buccal mucosa urethral replacement (Table 2).

Microscopic analysis under polarized light of histological slides stained by Picrosirius red was able to distinguish between collagen types I and III in the healing area of the implant. Table 3 presents the graft area occupied by total collagen and subtypes I and III. According to the data, there is clear predominance of type III collagen in all groups, regardless of the urethral substitute. However, type I collagen appeared in the same intensity in GI and GIII, but its presence was lower in the animals treated with four-layer SIS. For this reason, animals in GII had higher values during the calculation of collagen type III/I ratio.

\section{Discussion}

Several materials have been proposed for the reconstructive surgery of the urethra, such as synthetic tissues. However, clinical and experimental studies have shown poor success rates due to foreign body immunogenic reactions, ${ }^{11,12}$ erosion, fistulas, strictures, infections, urine extravasation, and encrustations. ${ }^{13,14}$

So, in an attempt to avoid these complications, more than 200 techniques of urethral reconstruction have been described, most of them using patient's penile shaft or foreskin pedicle flaps. ${ }^{15-17}$ However, when the local donor tissue is scarce or nonexistent, buccal or bladder mucosal grafts, lyophilized dura mater, saphenous vein, tunica vaginalis, 
Table 2. Mean and Standard Deviation of Graft Retraction Area in Square Millimeters, According to Different Methods of Analysis, in the Study Groups

\begin{tabular}{|c|c|c|c|c|}
\hline \multirow[b]{2}{*}{ Variable } & \multicolumn{3}{|c|}{ Groups } & \multirow[b]{2}{*}{ Statistics } \\
\hline & GI & GII & GIII & \\
\hline Elipses area & $21.52 \pm 1.07(b)$ & $16.16 \pm 5.25(\mathrm{ab})$ & $13.74 \pm 2.83(\mathrm{a})$ & $4.16(p<0.05)$ \\
\hline Digitalized area & $46.42 \pm 10.09(\mathrm{ab})$ & $58.48 \pm 18.16(\mathrm{~b})$ & $44.80 \pm 11.36(a)$ & $3.59(p<0.05)$ \\
\hline$\%$ Retraction & $55.27 \pm 9.68(\mathrm{ab})$ & $44.72 \pm 17.17(b)$ & $57.25 \pm 10.84(\mathrm{a})$ & $3.24(p<0.05)$ \\
\hline
\end{tabular}

intestinal mucosa, peritoneum, and total or partial skin grafts have been described in clinical and experimental trials. ${ }^{3,18-20}$ Of these materials, the buccal mucosa has been widely accepted as the gold standard for the treatment of lesions of the anterior urethra due to its durability and excellent results; however, mucosa graft success rates for repair of pendulous urethra were not so promising. $^{21,22}$

An alternative to traditional synthetic materials and tissue grafts currently used is based on the process of spontaneous regeneration of the urethra. Preliminary studies have demonstrated the efficacy of this method where the urethra is temporarily replaced by biocompatible and biodegradable materials showing regeneration and remodelation of its structure from the implanted tissue. ${ }^{1,23}$ One of the most promising materials for partial replacement of ureter, ${ }^{7,24}$ bladder $^{8,25-27,}$ and urethra, ${ }^{5,28-}$ 30 is SIS; a new collagen-based, nonimmunogenic, xenogenic material harvested from the submucosal layer of porcine intestine. The first reports on the use of SIS for urethra replacement were published by Atala and coworker ${ }^{31}$ to treat with hypospadias and insufficient penile skin for reconstruction.

The urethrocutaneous fistula is still a common complication in urethral surgeries. ${ }^{32,33}$ The use of SIS as an additional layer to cover the urethra was proposed by Grossklaus ${ }^{34}$ as an alternative to prevent fistulas. In our study, the incidence of fistula was the same in all groups, $8.3 \%$ (one animal per group), despite the fact that none of the animals remained with urinary diversion postoperatively. Sievert ${ }^{28}$ observed fistula in 4 of $24(16 \%)$ animals when the tubularized SIS graft was used to treat the urethra.

Partial urethral replacement by acellular collagen matrix has demonstrated adequate diameter at the urethrography. ${ }^{35}$ The replaced urethral segment showed a cellular organization close to the normal tissue, where retraction and stenosis were not observed. ${ }^{5}$ In our study, the analysis of urethral measurements performed 12 weeks after surgery did not show signs of stenosis or dilatation when compared with the preoperative retrograde urethrogram (RUG). Likewise, Atala ${ }^{5}$ observed no stenosis or signs of leakage urine at urethrography after surgery. Kropp, ${ }^{29}$ comparing SIS and preputial skin grafts, re- ported urethral diverticulum formation in $25 \%$ of patients treated with skin substitution resulting in more than $50 \%$ increase in the luminal area. However, none of the animals in the SIS group showed radiological changes of the urethra after 12 weeks of follow-up.

The SIS proved to be able to promote regeneration of transitional epithelium of bladder and urethral smooth muscle in rabbits. ${ }^{5,28-30}$ According to Kropp, ${ }^{25}$ there is a clear difference in the response of the host tissue to the graft foreskin when compared with SIS, where the latter provided a scaffold to the regenerative and remodeling process resulting in a normal urethral epithelium. In our study, all histological parameters evaluated were similar in the different groups. Histologically, at the end of the experiment, all animals had a graft recovered by a transitional cell epithelium from the urethral edges with a variable thickness from one to three layers of cells. The inflammatory infiltrate, composed of macrophages and polymorphonuclear cells, was mainly concentrated at the edges of the graft infiltrating the margins of the normal urethra. Similarly, we observed neovascularization characterized by delicate blood vessels composed of a thin wall, homogeneously distributed under the graft surface. Although others studies report minimal fibrosis with use of SIS, after 12 weeks, we observed extensive areas affected by fibrotic scar tissue characterized by an acellular eosinophilic infiltrate that extended under the edges of the normal urethral tissue. Atala, ${ }^{5}$ in a rabbit experimental model using SIS, reported that the membrane contained inflammatory cells derived from the host tissue, and the beginning of angiogenesis occurred about 2 weeks after surgery. As in our study, the author did not observe muscle regeneration after 2 weeks or 1 month of postoperative monitoring.

According to $\mathrm{Yao}^{36}$ collagen fibers types I and III are the main subtypes of fibers that influence the biological function of the urinary tract due to their biochemical characteristics and prevalence. Fibrotic tissues mostly exhibit increasing of collagen subtype I, considered more resistant and thicker than subtype III. ${ }^{37}$ In our model, we observed a higher collagen III/ I ratio in the animals treated with four-layer SIS. Similar to

Table 3. Median and Semi-Amplitude of the Graft Area, in Seuare Micrometers, Occupied by Total Collagen and Its Subtypes According to Study Group

\begin{tabular}{|c|c|c|c|c|}
\hline \multirow[b]{2}{*}{ Collagen } & \multicolumn{3}{|c|}{ Group } & \multirow[b]{2}{*}{ Statistics } \\
\hline & GI & GII & GIII & \\
\hline Type I & $302288.0 \pm 77494.0(b)$ & $1579.5 \pm 2503.8(\mathrm{a})$ & $18679.0 \pm 88662.0(\mathrm{~b})$ & $p<0.001$ \\
\hline Type III & $104792.5 \pm 153676.0(a)$ & $309872.9 \pm 252107.0(b)$ & $676647 \pm 893960.7(b)$ & $p<0.001$ \\
\hline Total & $978550.5 \pm 113933.0(b)$ & $820061.9 \pm 129278.5(a)$ & $789368.2 \pm 88661.5(\mathrm{a})$ & $p<0.001$ \\
\hline Ratio III/I & $119.68 \pm 32.26(\mathrm{a})$ & $257.23 \pm 180.12(b)$ & $115.09 \pm 154.72(a)$ & $p<0.01$ \\
\hline
\end{tabular}


that observed during the study of the graft area, these animals showed less tissue retraction, demonstrating that when the shrinkage is lower, there is a predominance of type III collagen fibers leading to less fibrosis.

Currently, there are a few papers comparing the results of SIS with one or four layers. We did not find significant differences in the histological parameters evaluated. Apparently, there is no variation in the response of host tissue to the urethral graft when we consider only the number of SIS layers. Soergel, ${ }^{10}$ in a retrospective study of twelve patients with severe hypospadias and chordee treated with four-layer SIS, reported a 33\% incidence of complications, higher than that observed with flaps of dermis and tunica vaginalis. Nuininga ${ }^{38}$ compared the results of the use of a single-layer SIS, four-layer SIS, and another type of collagen matrix obtained from bovine tendons in 24 rabbits submitted to partial urethral replacement. After 3 months, only the four-layer SIS was still distinguishable histologically, the urothelium had regenerated completely, with no signs of local inflammatory infiltrate. Hafez, ${ }^{39}$ evaluating 18 rabbits that had undergone penile chordee correction with SIS in one and four layers, noted that none of the animals in the first group had tissue retraction after 12 weeks of follow-up. On the other hand, four animals in the four-layer SIS group showed a $21 \%$ retraction after 6 weeks, and two of them showed a penile deviation of $15^{\circ}$ and $20^{\circ}$ during erection. In contrast with what was observed by Hafez, ${ }^{39}$ in our model, the computer analysis of tissue retraction showed that it was higher in the single-layer SIS group (55.27\%). Although the use of SIS (with one to four layers) or buccal mucosa as a urethral substitute is not new, this is the first study that confronts these two materials, evaluating their results in terms of histological, radiological, and morphometric aspects.

Tissue-engineering technology is promising in providing tissues that regenerate the urinary tract. The use of SIS is also promising, and reports have demonstrated this potential. However, some questions still need to be addressed, such as whether tissue regeneration can develop in diseased organs. This issue is particularly unclear in humans in whom the need to obtain cells from an abnormal tissue for cultivation or the use of a graft as a scaffold for the repair process of an impaired tissue could interfere with the final results. The understanding of the tissue regeneration process and the factors that influence the normal growth and cell function will assist us in obtaining the answer to such an important question.

\section{Conclusion}

The analysis of histological and imaging parameters showed no significant differences at the receptor urethral area in the three analyzed groups. However, analysis of the grafts area obtained from the computer program showed greater tissue retraction in the animals that had undergone urethral reconstruction with buccal mucosa and single-layer SIS. If we consider the advantages related to the physical properties of four-layer SIS, associated with lower tissue retraction (44.72\%) observed in our study, we can conclude that the four-layer SIS is more advantageous than the other two materials in the treatment of partial injuries of the urethra in the rabbit model.

\section{Disclosure Statement}

No competing financial interests exist.

\section{References}

1. Olsen L, Bowald S, Busch C, Carlsten J, Eriksson I. Urethral reconstruction with a new synthetic absorbable device. Scand J Urol Nephrol 1992;26:323-326.

2. Baskin LS, Duckett JW, Ueoka K, Seibold J, Snyder HM 3rd. Changing concepts of hypospadias curvature lead to more onlay island flap procedures. J Urol 1994;151:191-196.

3. Dessanti A, Rigamonti W, Merulla V, Falchetti D, Caccia G. Autologous buccal mucosa graft for hypospadias repair: An initial report. J Urol 1992;147:1081-1083.

4. McAninch JW. Reconstruction of extensive urethral strictures: Circular fasciocutaneous penile flaps. J Urol 1993;149: 488-491.

5. Chen F, Yoo JJ, Atala A. Acellular collagen matrix as a possible of the shelf biomaterial for urethral repair. Urology 1999;54:407-410.

6. Cheng EY, Kroop BP. Urologic tissue engineering with small intestinal submucosa: Potential clinical applications. World J Urol 2000;18:26-30.

7. Shalhav AL, Elbahnasy AM, Bercowsky E, Kovacs G, Brewer A, Maxwell KL, et al. Laparoscopic replacement of urinary tract segments using biodegradable materials in a large animal model. J Endourol 1999;13:241-244.

8. Kropp BP, Eppley BL, Prevel CD, Rippy MK, Harruff RC, Badylak SF, et al. Experimental assessment of small intestinal submucosa as a bladder wall substitute. Urology 1995; 46:396-400.

9. Sofer M, Rowe E, Forder DM, Denstedt JD. Ureteral segmental replacement using multilayer porcine small intestinal submucosa. J Endourol 2002;16:27-31.

10. Soergel TM, Cain MP, Kaefer M, Gitlin J, Casale AJ, Davis $\mathrm{MM}$, et al. Complications of small intestinal submucosa for corporal body grafting for proximal hypospadias. I Urol 2003;170:1577-1579.

11. Tsuji I, Kuroda K, Fujieda J, Shiraishi Y, Kunishima K. Clinical experiences of bladder reconstruction using preserved bladder and gelatin sponge bladder in the case of bladder cancer. J Urol 1967;98:91-92.

12. Taguchi H, Ishizuka E, Saito K. Cystoplasty by regeneration of the bladder. J Urol 1977;118:752-756.

13. Anwar H, Dave B, Seebode JJ. Replacement of partially resected canine urethra by polytetrafluoroethylene. Urology 1984;24:583-586.

14. Hakky SI. Urethral replacement by "Dacron" mesh. Lancet 1976;2:1192.

15. Turner-Warwick R. The repair of urethral strictures in the region of the membranous urethra. I Urol 1968;100:303-314.

16. Johanson B. Reconstruction of the male urethra in strictures: Application of the buried intact epithelium technique. Acta Chir Scand 1953;176:100-103.

17. Duckett JW. The island flap technique for hypospadias repair. Urol Clin North Am 1981;8:503-511.

18. Ransley PG, Duffy PG, Oesch IL, Van Oyen P, Hoover D. The use of bladder mucosa and combined bladder mucosa/ preputial skin grafts for urethral reconstruction. I Urol 1987;138:1096-1098.

19. Duckett JW, Coplen D, Ewalt D, Baskin LS. Buccal mucosal urethral replacement. J Urol 1995;153:1660-1663.

20. Ehrlich RM, Reda EF, Koyle MA, Kogan SJ, Levitt SB. Complications of bladder mucosal graft. J Urol 1989;142:626-627.

21. El-Kassaby A, Abou Shwareb T, Atala A. Randomized comparative study between buccal mucosal and acellular bladder matrix grafts in complex anterior urethral strictures. J Urol 2008;179:1432-1436. 
22. Bhargava S, Chapple CR. Buccal mucosal urethroplasty: Is it the new gold standard? BJU Int 2004;93:1191-1193.

23. Italiano G, Abatangelo G Jr., Calabrò A, Abatangelo G Sr., Zanoni $\mathrm{R}, \mathrm{O}^{\prime}$ Regan $\mathrm{M}$, et al. Reconstructive surgery of the urethra: A pilot study in the rabbit on the use of hyaluronan benzyl ester (Hyaff-11) biodegradable grafts. Urol Res 1997; 25:137-142.

24. Liatsikos EN, Dinlenc CZ, Kapoor R, Bernardo NO, Pikhasov $\mathrm{D}$, Anderson AE, et al. Ureteral reconstruction: Small intestine submucosa for the management of strictures and defects of the upper third of the ureter. J Urol 200;165:17191723.

25. Kropp BP, Rippy MK, Badylak SF, Adams MC, Keating MA, Rink RC, et al. Regenerative urinary bladder augmentation using small intestinal submucosa: Urodynamic and histopathologic assessment in long-term canine bladder augmentations. J Urol 1996;155:2098-2104.

26. Merguerian PA, Reddy PP, Barrieras DJ, Wilson GJ, Woodhouse K, Bagli DJ, et al. Acellular bladder matrix allografts in the regeneration of functional bladders: Evaluation of large-segment $\left(>24 \mathrm{~cm}^{2}\right)$ substitution in a porcine model. BJU Int 2000;85:894-898.

27. Kropp BP. Small intestinal submucosa for bladder augmentation: A review of preclinical studies. World J Urol 1998;16:262-267.

28. Sievert KD, Tanagho EA. Organ specific acellular matrix for reconstruction of the urinary tract. World J Urol 2000;18: $19-25$.

29. Kropp BP, Ludlow JK, Spicer D, Rippy MK, Badylak $\mathrm{SF}$, Adams $\mathrm{MC}$, et al. Rabbit urethral regenerate using small intestinal submucosa onlay grafts. Urology 1998;52: 138-142.

30. Chen F, Yoo JJ, Atala A. Experimental and clinical experience using tissue regeneration for urethral reconstruction. World J Urol 2000;18:67-70.

31. Atala A, Guzman L, Retik AB. A novel inert collagen matrix for hypospadias repair. J Urol 1999;162:1148-1151.

32. Goepel M, Otto T, Kröpfl D, Rübben H. Recent considerations for hypospadias repair: Results of 252 operations from 1985 to 1990. Eur Urol 1996;29:63-66.

33. Sorber M, Feitz WF, de Vries JD. Short and mid-term outcome of different types of one-stage hypospadias corrections. Eur Urol 1997;32:475-479.
34. Grossklaus DJ, Shappell SB, Adams MC, Brock JW 3rd, Pope JC 4th. Small intestinal submucosa as a urethral coverage layer. J Urol 2001;166:636-639.

35. Sievert K, Wefer J, Bakircioglu ME, Dahiya R, Herwig R, Hertle $\mathrm{L}$, et al. Reconstruction of the rabbit urethra with an acellular matrix (AUM) xenotransplantation. The Third World Congress on Urological Research, Paris, France, September 30-October 3, 1999.

36. Yao LY, Tekgu S, Kim KK, Li H, Mitchell ME, Carr MC. Developmental regulation of collagen differentiation expression in rabbit bladder. J Urol 1996;156:565-570.

37. Ewalt DH, Howard OS, Blyth B, Snyder HM, Duckett JW, Levin RM, et al. Is lamina propria matrix responsible for normal bladder compliance? J Urol 1992;148:544-549.

38. Nuininga JE, van Moerkerk $\mathrm{H}$, Hanssen A, Hulsbergen CA, Oosterwijk-Wakka J, Oosterwijk E, et al. Rabbit urethra replacement with a defined biomatrix or small intestinal submucosa. Eur Urol 2003;44:266-271.

39. Hafez AT, El-Assmy A, El-Hamid MA. 4 layer versus 1 layer small intestinal submucosa for correction of penile chordee: Experimental study in a rabbit model. I Urol 2004;171:24892491.

Address correspondence to: Paulo Roberto Kawano, M.D. Departamento de Urologia Universidade Estadual Paulista-UNESP Campus e Botucatu

Distrito de Rubião JR. s/n. Botucatu 18618970 Estado de São Paulo Brazil

E-mail: kawano@fmb.unesp.br

$\begin{aligned} & \text { Abbreviations Used } \\ \mathrm{GI} & =\text { group I } \\ \mathrm{GII} & =\text { group II } \\ \mathrm{GIII} & =\text { group III } \\ \mathrm{HE} & =\text { hematoxylin and eosin } \\ \mathrm{SIS} & =\text { small intestinal submucosa }\end{aligned}$

